\title{
Formação continuada de professores de Matemática com apoio de uma rede social na internet: mobilização de capital social
}

\author{
Gilmara T. Barcelos ${ }^{1}$, Liliana M. Passerino², Patricia A. Behar ${ }^{2}$ \\ ${ }^{1}$ Instituto Federal Fluminense Campus Campos-Centro \\ Rua Dr. Siqueira, 273. Parque Dom Bosco. CEP: 28030-130. Campos dos \\ Goytacazes/RJ \\ ${ }^{2}$ Universidade Federal do Rio Grande do Sul \\ Av. Paulo Gama 110. CEP: 90040-060. Porto Alegre/ RS \\ gilmarabeiff.edu.br, liliana@cinted.ufrgs.br, patricia.behar@ufrgs.br
}

\begin{abstract}
Information and communication technologies (ICT) have opened important possibilities for the learning of Mathematics. For this reason, it is important that teachers have the opportunity to be prepared. Considering that Math teachers education is a continuous process, the general objective of this thesis was to present a proposal for continuing education for former students of Mathematics Education, and to analyze the influence of such experience in their practice. The proposal is named T-PROIM - Tecnologias na Prática docente de pROfessores Iniciantes de Matemática (Technologies in the Teaching Practice of Beginning Teachers of Math). This aimed at enabling the integration of ICT into the teaching practice, and was based on the vygotskian theory. A qualitative research was carried through a case study in which data were collected from a questionnaire, interviews, observation and content posted on an internet social network (ISN). This article highlights the mobilization of the dimensions of social capital in interactions in the RSI. Data analysis showed the experience with T-PROIM influenced the subjects' teaching practice.
\end{abstract}

Resumo. As tecnologias de informação e comunicação (TIC) abrem importantes possibilidades para a aprendizagem de Matemática. Para tanto, é essencial que os professores tenham oportunidades para se prepararem. Considerando que a formação de professores de Matemática é um processo contínuo, o objetivo geral da pesquisa realizada foi construir e analisar uma proposta de formação continuada, para os egressos da licenciatura em Matemática, e, analisar a influencia dessa formação na prática docente. A proposta foi denominada T-PROIM - Tecnologias na Prática docente de pROfessores Iniciantes de Matemática. Essa visou a possibilitar a integração das TIC à prática docente e fundamentou-se na teoria vygotskyana. Uma Rede social na Internet, implementada com a plataforma Elgg, apoiou a formação. A pesquisa foi de caráter qualitativo por meio de um estudo de caso. As técnicas de coleta de dados foram questionário, entrevistas, observação e conteúdos postados na rede social na Internet (RSI). Neste artigo destaca-se a mobilização das dimensões do capital social nas interações na RSI. A análise dos dados sinalizou que a formação T-PROIM influenciou a prática docente dos professores quanto ao uso de TIC no processo de ensino e aprendizagem de Matemática. 


\section{Introdução}

O surgimento da Internet e, em especial das funcionalidades da Web 2.0 possibilitaram a criação de espaços de troca virtuais. Nesse contexto, com o suporte dos recursos Web 2.0 , as redes sociais ampliaram seus horizontes. Tais recursos possibilitaram uma nova forma de relacionamento, independente de tempo e espaço, por meio das chamadas redes sociais na Internet (RSI).

As RSI por meio das interações vêm modificando diversas áreas da atividade humana, a saber: comércio, indústria, economia, artes, cultura e educação, desde os mais remotos tempos da humanidade. Geralmente, as RSI são usadas para conhecer pessoas, disponibilizar fotos, vídeos, comentários, comercializar produto. Porém, também podem representar um importante recurso de apoio às atividades educacionais. Pesquisas recentes vêm mostrando que o uso de RSI, no contexto educacional, pode representar uma estratégia bastante interessante do ponto de vista pedagógico [Paião 2010]. Estas redes podem auxiliar programas de formação continuada de professores, possibilitando que estes vivenciem as vantagens das funcionalidades das RSI e assim tenham confiança para usá-las com seus alunos [Barcelos, Passerino e Behar 2010]. O professor em formação deve perceber as tecnologias como meio e possibilidade de ampliar os espaços educacionais, descentralizar o acesso ao saber, mudar a lógica de comunicação e aproximar professores e alunos.

Nesse sentido, é relevante repensar a formação dos professores para o uso de TIC. Segundo Costa (2008), a formação desses para integrar as TIC justifica-se caso corresponda a uma prática profissional melhor. A partir da percepção da necessidade de novas propostas de formação continuada ${ }^{1}$ e da compreensão das características das RSI elaborou-se uma proposta de formação continuada para professores iniciantes de Matemática. Esta foi denominada Tecnologias na Prática docente de pROfessores Iniciantes de Matemática (T-PROIM). Para apoiar as atividades presenciais e não presenciais, optou-se por utilizar a plataforma Elgg ${ }^{2}$, para a implementação da RSI. A gratuidade, o idioma, a variedade de dispositivos e o domínio dos recursos disponibilizados nas plataformas são aspectos considerados importantes para o contexto educacional e estão presentes na referida plataforma.

Nesse contexto, o objetivo deste trabalho é apresentar a mobilização das dimensões do capital social ocorrida no estudo de caso realizado na formação $T$ PROIM. Para tanto, na seção 2, são apresentadas reflexões sobre de redes sociais na internet e personal learning environments (PLE). A seguir, na seção 3, é descrito o contexto da pesquisa e os procedimentos metodológicos adotados. $\mathrm{Na}$ seção 4, é apresentada a análise dos dados levantados, destaca-se Finalizando, na seção 5, são feitas algumas considerações sobre o presente estudo.

\section{Redes Sociais na Internet e Personal Learning Environments}

\footnotetext{
${ }^{1}$ Propostas de formação padronizadas, ministrados por especialistas, composto de lições-modelo, nos quais o contexto dos professores participantes não é considerado, não tem modificado a prática docente. A formação docente deve superar a linearidade do processo educacional, de modo a possibilitar novas formas de ensinar, de aprender, de se organizar, de enxergar outras identidades sociais e manifestações culturais e de escutar outras vozes [IMBERNÓN 2010].

${ }^{2}$ Está disponível para download gratuito em: < http://www.elgg.org/download.php>.
} 
Uma rede social é definida como um conjunto de dois elementos: atores, ou seja, nós (pessoas, instituições ou grupos) e suas conexões (interações ou laços sociais) [Recuero 2009]. Os estudos sobre redes sociais não são novos, porém o enfoque deste modificouse com o tempo. Até o século XX, os cientistas estudavam as partes das redes, detalhadamente, visando, com isso, a compreender o todo. A partir do século XX, iniciaram-se estudos com foco nas interações entre as partes [Recuero 2009].

As formas de expressão numa rede social concretizam-se no estabelecimento de interações entre os nós da rede (atores da rede). Essas interações têm um caráter dinâmico e a análise das mesmas permite compreender que tipos de relacionamentos emergem na rede e que se constituem em laços sociais. As interações também possibilitam a mobilização de capital social. Segundo Putnam (1993), capital social refere-se à conexão entre indivíduos, são normas e valores que governam interações entre as pessoas. O "social" do capital social ressalta que esses recursos não são bens pessoais, e que residem em redes sociais. Warschauer (2006) ratifica afirmando que o capital social pode ser definido como a capacidade dos indivíduos de acumular benefícios por meio da força dos seus relacionamentos pessoais e da associação em redes e estruturas sociais específicas.

As RSI têm sido utilizadas para fins pedagógicos, porém na maioria das situações apenas como repositórios de materiais diversos. Não se questiona o valor dessas redes, mas considera-se que é possível ir além, criando laços mais fortes e num movimento dinâmico. Recuero (2010, p.1) ratifica afirmando que as RSI:

[...] podem e devem ser usados com propósitos educativos. Não é porque as pessoas "usam para conversar" que a ferramenta é uma "perda de tempo". A conversa faz parte do processo. A comunicação faz parte do processo. Proporcionar o uso dessas ferramentas, assim, pode ter resultados muito positivos em sala de aula. Estimular o uso criativo, a apropriação e a busca por mais informações são apenas algumas das maneiras de usar essas tecnologias.

O uso de RSI não é a solução para os problemas educacionais, mas pode contribuir para o processo de ensino e aprendizagem à medida que amplia as interações entre professores, entre alunos, entre professores e alunos e entre professores e pais de alunos. É importante ressaltar, porém, que o uso indevido dessas redes pode acarretar sérios problemas para os usuários, como por exemplo, o cyberbullying, a invasão de privacidade, o uso de perfis falsos, a divulgação de atitudes e conteúdos inapropriados, entre outros. Segundo Recuero (2010), muitos desses problemas acontecem devido à ausência de professores e pais nas RSI. Essa afirmação reforça a importância de professores conhecerem e analisarem os recursos disponíveis nas RSI.

Para a criação de RSI, faz-se necessário o uso de plataformas. Essas não são préconstruídas pelas plataformas, e, sim, apropriadas pelos atores sociais que lhes conferem sentido e que as adaptam as suas práticas sociais [Recuero 2012]. No âmbito deste trabalho, quatro plataformas foram analisadas, a saber: Facebook, Orkut, Ning e Elgg. Resumidamente, pode-se afirmar que o crescimento do uso do Orkut e do Facebook no Brasil e no mundo trouxe como consequências vários problemas como os já citados. Esses problemas fizeram com que os profissionais da educação (diretores, pedagogos, professores) bloqueassem o uso dessas plataformas, na maioria das escolas. A plataforma Ning disponibiliza excelentes recursos, possui uma interface amigável, porém a partir de agosto de 2010 deixou de ser gratuita ${ }^{3}$, fato que dificulta o uso da

\footnotetext{
${ }^{3}$ A gratuidade foi mantida apenas para a área de saúde e para educadores norte- americanos. Essa informação foi enviada por e-mail para todos os criadores de RSI na plataforma Ning.
} 
mesma no contexto educacional. Tais dificuldades sinalizaram que essas plataformas apresentam limitações para o uso educacional. Diante disto, buscaram-se plataformas de criação de redes sociais que superassem as limitações diagnosticadas e apresentassem características apropriadas para fins educacionais. Nesse sentido, foi encontrada a plataforma Elgg.

Alguns estudos sobre o uso da plataforma Elgg no contexto educacional já foram realizados (Garrett, et al., 2007; Tairi, et al., 2008; Ryberg, 2008; Dias, Oliveira, Alves, 2009) porém, não foi encontrado nenhum destinado à formação continuada de professores de Matemática visando a contribuir para a integração de tecnologias na prática docente ${ }^{4}$.

As mudanças sociais e culturais provocadas pelo desenvolvimento tecnológico, consequentemente, pela Web 2.0, além de contribuírem para o surgimento das RSI, também alavancaram o aparecimento dos personal learning environments ${ }^{5}$ (PLEs Ambientes pessoal de aprendizagem) [Mota 2009]. Os PLEs representam para a educação, os princípios do e-Learning 2.0, do poder e autonomia do utilizador, da abertura, da colaboração e da partilha, da aprendizagem permanente e ao longo da vida, da importância e valor da aprendizagem informal, das potencialidades dos softwares sociais, da rede como espaço de socialização, de conhecimento e de aprendizagem [Attwell 2007; Mota 2009]. A ideia de um PLE sustenta que a aprendizagem ocorre em diferentes contextos e situações, ampliando assim, o acesso à educação, por meio da possibilidade de inter-relacionar a aprendizagem da vida e a aprendizagem da escola ou da universidade [Attwell 2007]. Diante das características citadas, algumas plataformas de criação de RSI podem ser consideradas PLEs. Segundo Mota (2009), a plataforma Elgg, por exemplo, pode ser considerado um PLE, visualizando-a como um conjunto de ferramentas integradas (blog, perfil, rss, partilha de ficheiros, permissões finas, grupos, etc.) com grande controle por parte do utilizador. Na formação T-PROIM a plataforma Elgg foi usada na perspectiva de um PLE.

\section{Procedimentos metodológicos}

A pesquisa foi descritiva e explicativa, por meio de um estudo de caso e a abordagem, predominantemente, qualitativa. Foram utilizados os seguintes instrumentos de coletas de dados: observação, questionários, entrevistas e registro dos conteúdos postados na RSI pelos sujeitos participantes. Foram observados os comportamentos, os questionamentos e as ações dos participantes ao longo da formação T-PROIM na RSI e nos encontros presenciais. Os dados coletados foram analisados a partir da técnica análise de conteúdo [Bardin 2004]. Na análise, foram incluídos e cruzados os dados provenientes das diversas fontes e instrumentos.

As características da referida formação foram definidas a partir de um estudo de implementação que envolveu dois grupos de sujeitos: i) professores da licenciatura em Matemática em 2009, de um instituto federal de ensino (sujeitos do grupo 1); ii) professores de Matemática egressos da referida licenciatura que já estavam atuando na área (sujeitos do grupo 2). Diagnosticou-se, por meio da análise das respostas de um questionário, entre outros aspectos que os dezessete professores, sujeitos do grupo 1,

\footnotetext{
${ }^{4}$ Esse é o objetivo principal da pesquisa na qual o estudo descrito neste artigo está inserido.

${ }^{5} \mathrm{O}$ conceito de PLE é amplamente discutido na literatura, há uma diversidade de perspectivas e enfoques do que seja PLE. Segundo Attwell (2007), somente em uma coisa muitos autores concordam quanto ao PLE, não é um software, e sim, uma nova abordagem que usa tecnologias para a aprendizagem.
} 
conheciam e consideraram as TIC como importante recurso para o processo de ensino e aprendizagem. Porém, não usavam de forma integrada em suas práticas docentes. Considera-se que esse resultado não determina, mas influencia as práticas dos egressos dessa licenciatura. Os sujeitos do grupo 2, oito professores, afirmaram, por meio de uma entrevista, que a formação inicial preparou para o uso das TIC, porém as usavam muito pouco em suas práticas, fato que sinalizou que, entre outros fatores, a formação inicial não foi suficiente. A análise das entrevistas e da observação das práticas docentes desses sujeitos evidenciou que as aulas desses professores eram basicamente tradicionais e do tipo expositiva, com raras exceções. Além disso, diagnosticou-se que os egressos necessitavam de uma formação continuada que: i) fundamentasse e orientasse a utilização das TIC nas escolas; ii) considere o contexto do professor nas atividades; iii) criasse espaço de elaboração de recursos pedagógicos e de publicação; iv) proporcionasse momentos de formação entre pares [Barcelos 2011].

Além disso, foi realizado um minicurso com a participação de dezoito professores em formação e em serviço, visando a analisar o uso dos recursos da plataforma Elgg. A análise das atividades realizadas no minicurso e das respostas de um questionário sinalizou que os recursos experimentados na plataforma Elgg são fáceis de usar e contribuem para a interação entre professor e aluno e entre os alunos.

Todo o contexto descrito possibilitou levantar características e definir os objetivos da formação T-PROIM a qual considerou a formação inicial dos participantes, criando um elo entre a universidade e sala aula, para além dos estágios supervisionados. Os objetivos da formação foram: i) fundamentar a utilização das TIC nas escolas nas quais os professores/participantes atuam; ii) considerar o contexto do professor nas atividades; iii) criar espaço de criação de recursos pedagógicos e de publicação dos mesmos; iv) proporcionar momentos de formação entre pares.

Uma RSI implementada com plataforma Elgg apoiou e orientou os professores em momentos presenciais e não presenciais de forma a garantir maior participação. Para uso dessa plataforma foi necessário instalar, configurar e organizar todos os recursos que seriam convenientes para essa pesquisa. Assim como criar um logotipo e customizar as páginas. As atividades propostas foram realizadas considerando o contexto da sala de aula de cada professor, tentando assim minimizar o problema da não aplicação do que é estudado nos programas de formação continuada, nas atividades docentes. Essa rede, denominada T-PROIM, mesmo nome da formação, foi considerada um PLE. O públicoalvo foi composto por oito egressos da licenciatura em Matemática de um instituto federal, que estavam iniciando suas práticas docentes (professores iniciantes). O período estabelecido para ocorrer a formação T-PROIM fundamenta-se no fato de que as bases dos saberes profissionais, geralmente, são construídas no início da carreira [Tardif 2007]. Além de ser o início uma fase crítica, muitas vezes, decorrente do confronto inicial com a dura e complexa realidade do exercício da profissão [Tardif 2007]. Ocorreram dez encontros presenciais (três horas cada), uma palestra online e muitas interações por meio da RSI.

O processo de formação foi elaborado a partir da concepção epistemológica sócio-histórica e por esse motivo considera as interações interpessoais como etapa essencial para a internalização dos processos psicológicos superiores [Vygotsky 2007]. Todo o processo de formação foi baseado na realização de atividades práticas, estas sempre fundamentadas em teorias educacionais, a relação teoria e prática foi 
integradora.

Partindo da premissa que as relações e interações ocorridas na formação $\mathrm{T}$ PROIM são fundamentais para o uso das TIC na prática docente dos professores participantes da formação estabeleceu-se que as interações seriam a categoria inicial de análise para a experimentação da formação T-PROIM. A seguir, para fins de observação e análise das relações e interações numa visão sócio-histórica estabeleceram-se os seguintes indicadores: i) nível de participação: ativa, reativa ou passiva. Ativa quando o sujeito participa voluntariamente da atividade sem intervenção do mediador; reativa, quando o sujeito participa sempre que o mediador solicita e passiva quando não participa, ficando apenas observando a ação da mediadora [Passerino 2005]; ii) práticas de letramento: por meio do computador, informacional, multimídia e comunicacional [Warschauer 2006]; iii) nível de integração das TIC: não-utilização; tomada de consciência; exploração; infusão; integração mecânica; integração rotineira; expansão; refinamento [Moersch 1998]; iv) dimensões do capital social: relacional, cognitivo, normativo, de confiança no ambiente, institucional [Bertolini e Bravo 2004]. A partir dessa análise, foram identificados os estágios de uma comunidade de prática que os participantes da formação T-PROIM vivenciaram, segundo estudos de Wenger; McDermott e Snyder (2002): potencial, coalescente, maturação, hospedagem e transformação [Barcelos 2011]. Neste artigo destaca-se a análise da mobilização das dimensões do capital social a partir das interações ocorridas na formação T-PROIM.

\section{Análise e discussão dos resultados}

Uma análise macro foi realizada visando a identificar o contexto e possíveis dificuldades de uso da plataforma Elgg, pois era desejável que as referidas dificuldades não interferissem na qualidade dos conteúdos postados e das interações.

As atividades presenciais e por meio da RSI possibilitaram a mobilização de todas as dimensões do capital social proposta por Bertolini e Bravo (2004): relacional CSR; cognitivo- CSC; normativo - CSN; de confiança no ambiente social- CSCA e institucional - CSI. A valorização, em igual proporção das cinco dimensões do capital social, incentivou a participação ativa e embasou iniciativas importantes de uso pedagógico das TIC.

Capital social relacional (CSR) corresponde à soma das relações sociais, laços e trocas que conectam os indivíduos de uma determinada rede, em nível individual ou coletivo [Bertolini e Bravo 2004]. Essa dimensão do capital possiblita a exposição do cotidiando, do contexto familiar, de valores e crenças. Essa dimensão do capital social foi identificada em várias mensagens postadas no microblog. As professoras 5 e 8, por exemplo, desejaram a todos os membros da rede, "Feliz Páscoa". Essa ação foi o ponto de partida para trocas sociais dessa natureza durante toda a formação. Destaca-se que esse capital social mobilizado é importante para o contexto educacional, por considerar que a formação vai além do aspecto cognitivo. Características culturais e sociais revelam o contexto social, aspectos considerados importante na teoria sócio-histórica. Segundo Vygotsky (2007), o movimento de mudança individual, ao longo do seu desenvolvimento, tem raiz na sociedade e na cultura; a interação entre os indivíduos desempenha um papel fundamental na construção do ser humano.

Expressar sentimentos, problemas e situações cotidianas para os demais membros da RSI foi uma ação realizada, intensamente, por meio do microblog. Tudo 
isso mobilizou CSR, pois, laços eram estabelecidos por meio dos sentimentos expressos. A abertura possibilitada pelo microblog permite aos usuários, se expressarem para além do conteúdo em estudo. Mensagens desse tipo retratam que os professores entenderam que a estrutura da rede não é linear e que não há um lugar único na rede para tais postagens. Esse fato sinaliza que a RSI da formação T-PROIM pode ser vista como um PLE. O diálogo informal, ocorrido pelos diversos recursos da RSI, é importante para apoiar o desenvolvimento de atividades docentes e possibilita a mobilização de CSR.

Capital social cognitivo (CSC) é a soma dos conhecimentos e das informações compartilhadas por um determinado grupo [Bertolini e Bravo 2004]. Relaciona-se com a transmissão, aquisição e construção de conhecimentos. Esse capital foi mobilizado, predominantemente, na formação T-PROIM. Atribui-se esse fato a ser essa a dimensão do capital social que os professores, durante toda sua formação, mais vivenciaram e que, provavelmente, buscaram na formação continuada.

Desde o primeiro encontro presencial (17 de março de 2011), foi comunicado aos participantes que a formação T-PROIM era um espaço de troca de experiências e conhecimentos e também de apoio à prática docente. Pelas postagens na RSI, já no mês de março, foi possível perceber as primeiras trocas. Essas postagens evidenciam CSC (conteúdo das listas de exercícios e a análise da resolução da lista de exercício, por meio dos comentários). A prática de postagem de atividades e materiais utilizados no contexto docente ocorreu em todo o período da formação T-PROIM. Destaca-se que as atividades ou provas postadas sempre continham figuras construídas pelas professoras, com auxílio de alguma tecnologia e, além disso, eram sempre bem arrumadas. De maneira geral, os links compartilhados em favoritos mobilizaram CSC, pois sempre conduziam a algo novo que alguém descobria e compartilhava com os demais (endereços de softwares estudados ou citados nas discussões orais, blogs sobre temas em estudo, livro, vídeos, sites contendo materiais pedagógicos, entre outros).

Uma prática contínua foi a criação de blog na RSI da formação, a partir da postagem de textos a qual gerou comentários ricos em CSC à medida que os professores apresentavam ideias além das apresentadas no texto. A P1, por exemplo, afirmou que ficou surpresa ao ler sobre o uso educacional do Twitter em um dos textos do blog da rede, porém destaca que muitas são as dificuldades a serem enfrentadas para que as TIC sejam integradas ao contexto escolar, embora já perceba progressos. Os diversos temas discutidos na formação levaram a P7 postar, em favoritos, o link de um vídeo que retratava o uso de tecnologias, em particular, o uso de celular e de redes sociais. Essa postagem sinaliza a influência da formação, à medida que retoma temas debatidos. Os comentários do link postado sinalizaram a relação horizontal existente entre a mediadora e a professora o que facilita as interações. Além disso, revelou a percepção de que os temas abordados na formação T-PROIM são atuais.

Capital social normativo (CSN) é um conjunto de normas de comportamento de um determinado grupo e valores desse grupo [Bertolini e Bravo 2004]. Essa dimensão do capital social, assim como as outras foram mobilizadas em diversas situações. O uso do microblog para descrever o que está sendo feito foi uma prática comum, destaca-se que o referido recurso foi usado para comunicar o cumprimento de uma atividade solicitada na formação, ou seja, CSN. A presença nos encontros presenciais era uma exigência da formação (norma), a P6 usou o microblog para avisar que a sua seria virtual num determinado encontro. Já P2 usou o mesmo recurso para 
justificar sua falta. Em ambos os casos CSN foi mobilizado, afinal estavam explicando o não cumprimento de uma norma da formação, a presença. A participação na RSI da formação também pode ser considerada uma norma, afinal parte das atividades eram realizadas por meio da mesma. Considera-se que troca de CSN foi muito importante para os professores da formação T-PROIM, já que essa dimensão do capital social deve ser mobilizada também, nas práticas docentes dos mesmos, como forma de organizar as atividades a serem desenvolvidas com seus alunos.

Capital social de confiança no ambiente social (CSCA) refere-se ao nível de confiança que o indivíduo deposita em um determinado ambiente social [Bertolini e Bravo 2004]. Um elevado grau de confiança no ambiente social permite uma redução da incerteza em face das ações possíveis e a vontade de cooperar dos outros membros.

Uma das atividades solicitadas foi a escolha de um objeto de aprendizagem que contemplasse algum conteúdo do planejamento anual. Esse objeto deveria ser apresentado para os demais professores no encontro presencial. Porém, todos os professores tiveram dificuldades de acessar os repositórios sugeridos. O microblog da rede foi usado por P7 para partilhar essa dificuldade, a mediadora tentou ajudar por meio do mesmo recurso. Considera-se que a manifestação da dificuldade por meio da RSI mobiliza CSCA e já as trocas de informação mobilizam CSC e CSR. Os objetos de aprendizagem foram apresentados e discutidos produtivamente. CSCA foi mobilizado em praticamente todas as postagens, afinal tudo que é compartilhado na RSI ou mesmo oralmente (registrado no diário de campo) reflete confiança no ambiente da formação TPROIM. Os professores compartilhavam muito mais do que foi solicitado pela pesquisadora, o que engrandeceu as trocas e fortificou os laços sociais.

Capital social institucional (CSI) relaciona-se com a instituição formal ou informal em que o grupo social está inserido [Bertolini e Bravo, 2004].

Como identificado, por meio do questionário e da entrevista, a infraestrutura das escolas quanto a tecnologias não era tão boa, sendo assim outros recursos eram usados no desenvolvimento das aulas. No recurso álbum de fotos da RSI P4, P5 e P8 disponibilizaram fotos de atividades realizadas com materiais concretos As atividades eram descritas e as fotos disponibilizadas. Essa ação possibilitou, de forma singela, a troca de CSI, afinal as atividades eram relacionadas à instituição em que atuavam. Além disso, por revelar que o material concreto é algo acessível às instituições, o que nem sempre acontece com as tecnologias. CSC também foi mobilizado, por meio da descrição das atividades desenvolvidas (troca de estratégias) e pelos conteúdos abordados pelas atividades.

O que há de comum em todas as situações apresentadas é que, em geral, mobilizaram diferentes dimensões do capital social [Bertolini e Bravo 2004] porém, focalizaram-se as dimensões predominantes. As diversas formas de troca de capital social foram possíveis devido aos recursos disponíveis na RSI, criada com a plataforma Elgg. Esses ampliam os espaços de interação entre os participantes dos processo permitindo maior participação. Considera-se que o capital social gerado em toda a formação T-PROIM possibilitou os professores participantes à construção de conhecimentos. Conhecimentos que direta ou indiretamente influenciaram na prática docente dos mesmos.

\section{Considerações Finais}


Durante toda a formação T-PROIM, a participação não foi sempre na mesma intensidade tanto nos encontros presenciais quanto na RSI. Porém, foi possível perceber que sempre que possível passavam pela RSI para olhar as novidades ("Passando para olhar as novidades" - P8, "Vendo as novidades"- P7), ou para compartilhá-las. Considera-se que postagens dessa natureza incentivam outras interações, à medida que tanto a mediadora quanto os professores sempre usavam a rede para postar novidades, incrementando os laços, transformando laços fracos em fortes e mobilizando CSR.

Postagens semelhantes a essas foram feitas após o último encontro presencial. Esse fato revela a importância da rede para essas professoras.

Na formação T-PROIM vivenciou-se que a RSI pode ser considerada um PLE e, além disso, quando essa rede é incorporada pelos usuários/produtores, contribui para a constituição de uma CP e influencia a prática docente. A rede também possibilitou atender às necessidades de uma formação continuada. A descentralização dos conteúdos, o controle por parte de todos os usuários, a valorização de contribuições espontâneas (autonomia e criatividade) e o saber lidar com os problemas recorrentes de toda flexibilidade constituem um desafio. A mobilização das diversas dimensões do capital social enriqueceu todo o processo de formação.

Essa pesquisa não tentou criar uma relação direta entre a incorporação das TIC e as aprendizagens, uma vez que essa relação está associada a um amplo e complexo leque de fatores que constituem as práticas educacionais. Limitou-se a contribuir com práticas docentes mais inovadoras e que incentivem os alunos a aprenderem a aprender com o apoio da RSI o que, diante do contexto dos professores participantes da pesquisa, foi considerado um grande avanço.

\section{Referências}

Attwell, G. (2007). Personal Learning Environments - the future of eLearning? Elearningpaper, v.2, n. 1, Jan.2007.

Barcelos, G. T., Passerino, L. M. e Behar, P. A. (2010). Análise dos Impactos da Integração de Tecnologias na Formação Inicial de Professores de Matemática sobre a prática docente: um estudo de caso. In: Workshop de Informática na Escola (WIE), 15, Belo Horizonte, MG, SBC.

Barcelos, G. T. (2011). Tecnologias na prática docente de professores de Matemática: formação continuada com apoio de uma rede social na internet. Tese. (Doutorado em Informática na Educação). Universidade Federal do Rio Grande do Sul - UFRGS, Porto Alegre, RS.

Bardin, L. (2004). Análise de conteúdo. Tradução de Luís Antero Reto e Augusto Pinheiro. 3.ed. Lisboa-Portugal: Edições 70.

Bertolini, S.; Bravo, G. (2004). Social Capital, a Multidimensional Concept. 2004. Disponívelem:<http://web.archive.org/web/20030318075349/http://www.ex.ac.uk/shi pss/politics/research/socialcapital/other/bertolini.pdf>. Acesso em: 23 jul. 2012.

Costa, F. Coord. (2008). Competências TIC: estudo de Implementação, v.1. Lisboa: GEPE/ME (Gabinete de Estatística e Planejamento da Educação).

Dias, C. M. V.; Oliveira, L . R. M. e Alves, M. P. C. (2009), Recognition, Validation And Certification Of Competences Using Eportfolio. Contributions to Changing the Evaluation Paradigm and to the Development of Computer Science Literacy.

Garrett, N.; Thoms, B.; Soffer, M. e Ryan, T. (2007) Extending the Elgg Social 
Networking System to Enhance the Campus Conversation. Second Annual Design Research in Information Systems (DESRIST), Pasadena, California, 14-15 May.

Imbernón, F. (2010). Formação Continuada de Professores. Tradução de Juliana dos Santos Padilha. Porto Alegre: Artmed.

Moersch, C. (1998). Computer efficiency: measuring the instructional use of technology. Learning and Leading With Technology, December/January 19961997. ISTE - International Society for Technology in Education, p. 52- 56, 1998.

Moreira, J. A. M. e Monteiro, A. A. (2010). O trabalho pedagógico em cenários presenciais e virtuais no ensino superior. Educação, Formação \& Tecnologias, v.3, n. 2, p. 82-94.

Mota, J. (2009). Personal Learning Environments: contributos para uma discussão do conceito. Educação, Formação \& Tecnologias, v.2, n.2, pp. 5-21, Nov. de 2009.

Paião, C. (2010). Plataformas sociais auxiliam a construção do conhecimento? Com Ciência: revista eletrônica de jornalismo científico, 121.

Passerino, L. M. (2005). Pessoas com autismo em ambientes digitais de aprendizagem: estudo dos processos de interação social e mediação. Tese. (Doutorado em Informática na Educação). Universidade Federal do Rio Grande do Sul - UFRGS, Porto Alegre, RS.

Recuero, R. (2009). Redes sociais na Internet. Porto Alegre, RS: Sulina.

Recuero, R. (2010). Sites de Redes Sociais e Educação. Disponível em:< http://www.pontomidia.com.br/raquel/arquivos/2010/07/sites-de-redes-3.html >. Acesso em: 14 ago. 2012.

Recuero, R. (2012). A conversação em rede: comunicação mediada pelo computador e redes sociais na internet. Porto Alegre, RS: Sulina.

Ryberg, T. (2008). Privacy, power, place and identity - the construction of mixed spaces in an educational context. Paper presented at Internet Research 9.0: Rethinking Community, Rethinking Place, København, Denmark.

Tairi, K., Mccormack, R., Leihy, P., \& Ring, P. (2008). Fairy tales and Elggs: social networking with student rovers in learning commons. Proceedings of the VALA 14th Biennial Conference and Exhibition, 05-07, Melbourne, Australia.

Tardif, M. (2007). Saberes Docentes e Formação Profissional. 8. ed. Tradução de Francisco Pereira. Petrópolis-RJ: Vozes.

Vygotsky, L. S. (2007). A formação social da mente: o desenvolvimento dos processos psicológicos superiores. 7. ed. São Paulo: Martins Fontes.

Wajskop, G (Col.) (2009). A formação e a iniciação profissional e as implicações sobre a qualidade do ensino. Sâo Paulo: Instituto de Evaluación y Asesoramiento Educativo.

Warschauer, M. (2006). Tecnologia e inclusão social: a exclusão digital em debate. Tradução Carlos Szlak. São Paulo: Editora Senac.

Wenger, E.; McDermott, R. e Snyder, W. M. (2002). Cultivating communities of practice: a guide to managing knowledge. Boston: Harvard Business School Press. 